\title{
Evaluationsbogen
}

\section{Septischer Schock und systemisches Entzündungsreaktions-Syndrom}

Angaben zur Person

Name, Vorname,Titel:

Straße, Hausnr.: PLZ/Ort:

Ich bin Mitglied der Ärztekammer (bitte Namen der Kammer eintragen): Anschrift: $\square$ privat $\square$ dienstlich Jahr meiner Approbation:

Ich befinde mich in der Weiterbildung zum:

Ich habe eine abgeschlossene Weiterbildung in (bitte Fach eintragen):

Ich bin tätig als: $\square$ Assistenzarzt $\square$ Oberarzt $\square$ Chefarzt $\square$ niedergelassener Arzt $\square$ Sonstiges:

Ich bin DMV-Abonnent: $\square$ ja $\square$ nein

Falls nein: ich habe den Fragebogen aus/von: $\square$ Thieme-connect $\square$ Kollegen $\square$ der Klinik $\square$ einer Bibliothek $\square$ Sonstiges

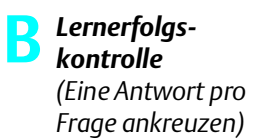
$1 \mathrm{~A}, \mathrm{~B}, \mathrm{C}, \mathrm{D}, \mathrm{E}$
$7 \mathrm{~A}, \mathrm{~B}, \mathrm{C} \mathbb{E}$
$2 \mathrm{~A}[\mathrm{~B}[\mathrm{C} \in \mathbb{D}[\mathrm{E}$
8 A B C $\mathbb{D}$ D
3 A $B \in[C, D[E$

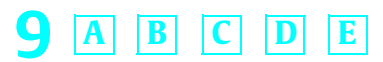
$4 \mathrm{~A} B \mathrm{~B}$ D $\mathrm{E}$
10 A $B$ C $\mathrm{D}$ E
5 A $B$ C $\mathrm{C}$ D $\mathrm{E}$
$11 \mathrm{~A}, \mathrm{C}, \mathrm{D}$
$6 \mathrm{~A}, \mathrm{~B}, \mathrm{D}, \mathrm{E}$

Erklärung

Ich versichere, dass ich die Beantwortung der Fragen selbst und ohne fremde Hilfe durchgeführt habe

Ort/Datum: Unterschrift

Feld für CMEWertmarke Wertmarke $\mathrm{ChO}$
Bitte in dieses Feld die CME-Wertmarke kleben oder Ihre DMW Abonnement-Nummer eintragen: (siehe Adressaufkleber Ihrer DMW)

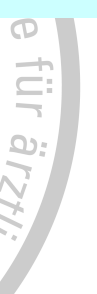

Zertifizierungsfeld (wird durch die DMW ausgefüllt)

\section{Ihr Ergebnis}

Sie haben von 11 Fragen richtig beantwortet. Im Durchschnitt wurden __ der Fragen richtig beantwortet. Sie haben $\square$ bestanden $\square$ nicht bestanden. 口ungültig, weil:

Stuttgart, den Datum Stempel/Unterschrift 
Fragen zur Zertifizierung (bitte unbedingt ausfüllen bzw. ankreuzen, da Evaluation sonst unvollständig!)

\section{Didaktisch-methodische Evaluation}

1 Septischer Schock und SIRS kommen in meiner ärztlichen Tätigkeit

$\square$ häufig vor

$\square$ regelmäßig vor

$\square$ selten vor

$\square$ gar nicht vor

2

Bezüglich der Diagnostik und Therapie von septischem Schock und SIRS gab es für mich vor dem Studium dieses Beitrages

$\square$ eine feste Gesamtstrategie

$\square$ noch offene Einzelprobleme:

$\square$ keine Strategie

3

Bezüglich septischem Schock und SIRS

$\square$ fühle ich mich nach dem Studium des Beitrages in meiner Strategie bestätigt

$\square$ habe ich meine Strategie verändert:

$\square$ habe ich erstmals eine einheitliche Strategie erarbeitet

$\square$ habe ich keine einheitliche Strategie ableiten können

4.

Wurden aus der Sicht Ihrer täglichen Praxis heraus wichtige Aspekte des Themas

a) nicht erwähnt? $\square$ ja $\square$ nein

Wenn ja, welche:

b) zu knapp abgehandelt? $\square$ ja $\square$ nein

Wenn ja, welche:

c) überbewertet? $\square$ ja $\square$ nein

Wenn ja, welche:

5

Verständlichkeit des Beitrages

$\square$ Der Beitrag ist nur für Spezialisten verständlich

$\square$ Der Beitrag ist auch für Nicht-Spezialisten verständlich

Beantwortung der Fragen

$\square$ Die Fragen lassen sich aus dem Studium des Beitrages allein beantworten

$\square$ Die Fragen lassen sich nur unter Zuhilfenahme zusätzlicher Literatur beantworten

Die Aussagen des Beitrages benötigen eine ausführlichere Darstellung

$\square$ zusätzlicher Daten

$\square$ von Befunden bildgebender Verfahren

$\square$ die Darstellung ist ausreichend 